\title{
Sepsis results in early cholesterol and steroidogenesis pathway alterations
}

\author{
W Khaliq ${ }^{1 *}$, DT Andreis ${ }^{1,2}$, M Singer $^{1}$ \\ From ESICM LIVES 2015 \\ Berlin, Germany. 3-7 October 2015
}

\section{Introduction}

Both steroid and sex hormones regulate multiple physiological functions including the stress response, fluid retention, metabolism and reproduction. They are synthesized from cholesterol via the precursor steroid pregnenolone. We previously reported early falls in HDL and LDL cholesterol levels in a fluid-resuscitated rat model of faecal peritonitis [1] and these could prognosticate as early as 6 $\mathrm{h}$ post-insult [2]. Pregnenolone may be converted to either (i) progesterone, corticosterone and then aldosterone via a common pathway or (ii) to dehydroepiandrosterone (DHEA), the precursor to testosterone and estradiol.

\section{Objectives}

Using our $72 \mathrm{~h}$ rat model of faecal peritonitis, we sought to investigate the relationship between hypocholesterolaemia and abnormalities in the steroidogenesis pathways in sepsis.

\section{Methods}

Awake, instrumented yet fully mobile male Wistar rats $(325 \pm 15 \mathrm{~g})$ received an i.p. injection of $4 \mu \mathrm{l} / \mathrm{g}$ faecal slurry.
Fluid resuscitation (50:50 mix of 5\% glucose/Hartmann's; $10 \mathrm{ml} / \mathrm{kg} / \mathrm{h}$ ) was commenced at $2 \mathrm{~h}$. At $6 \mathrm{~h}$, an echo-measured heart rate cut-off of $460 \mathrm{bpm}$ was used to classify animals into predicted survivors or non-survivors. Blood samples were taken concurrently for measurement of the lipid profile (enzymatic colorimetric analysis) and plasma levels of progresterone, corticosterone, aldosterone, DHEA, testosterone and estradiol (ELISA). Control animals were treated identically except for slurry injection. Results were analysed using two-way ANOVA and posthoc testing and considered statistically significant when $\mathrm{p}<0.05$.

\section{Results}

At $6 \mathrm{~h}$ septic animals had significant hypocholesterolaemia, the magnitude of which was significantly greater in predicted non-survivors. Levels in the progesteronecorticosterone-aldosterone steroidogenesis pathway were all elevated ( $\mathrm{p}<0.05$ vs controls), whereas DHEAtestosterone-estradiol steroidogenesis pathway levels were all subnormal (Table 1).

Table 1

\begin{tabular}{|c|c|c|c|}
\hline & Control $(n=6)$ & Predicted survival $(n=6)$ & Predicted non-survival $(n=6)$ \\
\hline HDL cholesterol (mmol/L) & $1.03 \pm 0.07$ & $0.88 \pm 0.04^{a}$ & $0.73 \pm 0.07^{\mathrm{a}, \mathrm{b}}$ \\
\hline Progesterone $(\mathrm{ng} / \mathrm{mL})$ & $31.1 \pm 3.5$ & $38.3 \pm 5.2$ & $41.6 \pm 3.7^{a}$ \\
\hline Corticosterone $(\mathrm{pg} / \mathrm{mL})$ & $231 \pm 36$ & $661 \pm 121^{\text {a }}$ & $719 \pm 87^{a}$ \\
\hline Aldosterone $(\mathrm{pg} / \mathrm{mL})$ & $86 \pm 8$ & $172 \pm 24^{a}$ & $619 \pm 109^{a, b}$ \\
\hline DHEA $(\mathrm{pg} / \mathrm{mL})$ & $138 \pm 13$ & $109 \pm 21^{a}$ & $66 \pm 13^{a, b}$ \\
\hline Testosterone (pg/mL) & $156 \pm 14$ & $107 \pm 8^{a}$ & $100 \pm 7^{a}$ \\
\hline 17ß-Estradiol $(\mathrm{pg} / \mathrm{mL})$ & $84 \pm 6$ & $71 \pm 8$ & $69 \pm 9$ \\
\hline
\end{tabular}

${ }^{1}$ University College London, Bloomsbury Institute of Intensive Care Medicine, London, United Kingdom 


\section{Conclusions}

Sepsis resulted in significant early reductions in cholesterol levels. This reduction may be due to increased cholesterol utilization for preferential steroidogenesis in the progesterone-corticosterone-aldosterone pathway, at the expense of DHEA-testosterone-estradiol steroidogenesis. This relationship has not, to our knowledge, been previously described in sepsis and warrants further investigation.

\section{Grant Acknowledgment}

ESICM Basic Science Award, Intensive Care Foundation (UK), NIHR

\section{Authors' details}

${ }^{1}$ University College London, Bloomsbury Institute of Intensive Care Medicine, London, United Kingdom. ${ }^{2}$ Università degli Studi di Milano, Dipartimento di Fisiopatologia Medico-Chirurgica e dei Trapianti, Milan, Italy.

Published: 1 October 2015

\section{Reference}

1. Khaliq W, et al: Intensive Care Med Experimental 2014, 2(S1):22.

doi:10.1186/2197-425X-3-S1-A978

Cite this article as: Khaliq et al:: Sepsis results in early cholesterol and steroidogenesis pathway alterations. Intensive Care Medicine Experimental 2015 3(Suppl 1):A978.

\section{Submit your manuscript to a SpringerOpen ${ }^{\mathcal{O}}$ journal and benefit from:}

- Convenient online submission

- Rigorous peer review

- Immediate publication on acceptance

- Open access: articles freely available online

- High visibility within the field

- Retaining the copyright to your article 